\title{
Non-Recurrent Right Laryngeal Nerve: a Rare Anatomic Variation Encountered During a Total Thyroidectomy
}

\author{
Dimosthenis Chrysikos ${ }^{1, *}$, Markos Sgantzos², John Tsiaoussis 3 , George Noussios 4 , Theodore Troupis ${ }^{5}$, \\ Vassilios Protogerou ${ }^{5}$, Eleftherios Spartalis ${ }^{6}$, Tania Triantafyllou ${ }^{7}$, Theodoros Mariolis-Sapsakos ${ }^{1}$
}

\section{ABSTRACT}

The non-recurrent laryngeal nerve (nRLN) is a rare anatomic variation that every head and neck surgeon must be aware of, in order to avoid intraoperative injury which leads to postoperative morbidity.

We are reporting a case of a nRLN in a 47 year old female patient with medullary thyroid carcinoma who was surgically treated with total thyroidectomy and lymph node dissection. Both two inferior laryngeal nerves were identified, fully exposed and preserved along their cervical courses. However, we found that the right inferior laryngeal nerve was non-recurrent and directly arised from the cervical vagal trunk, entered the larynx after a short transverse course and parallel to the inferior thyroid artery.

The safety of thyroid operations is dependent on high index of suspicion, meticulous identification and dissection of laryngeal nerves either recurrent or non-recurrent. This leads to minimum risk of iatrogenic damage of the nerves.

Complete knowledge of the anatomy of these neural structures, including all their anatomic variations is of paramount importance.

\section{KEYWORDS}

non-recurrent laryngeal nerve; thyroidectomy; vagus nerve

\section{AUTHOR AFFILIATIONS}

${ }^{1}$ University Department of Surgery, General and Oncologic Hospital of Kifissia "Agii Anargiri", Athens, Greece

2 Department of Anatomy, Medical School, University of Thessaly, Larissa, Greece

${ }^{3}$ Department of Anatomy, School of Medicine, University of Crete, Greece

${ }^{4}$ Department of Physical Education and Sports Sciences, "Aristotles", University of Thessaloniki, Serres, Greece

${ }^{5}$ Faculty of Medicine Department of Anatomy National and Kapodistrian University of Athens Athens Greece

${ }^{6}$ Laboratory of Experimental Surgery and Surgical Research, University of Athens Medical School, Athens, Greece

7 1st Propaedeutic Surgical Clinic, Hippocration General Hospital Athens, Greece

* Corresponding author: Dimosthenis Chrysikos, Gortynias 9, Athens, Greece Post Code: 16561; e-mail: dixrys@yahoo.gr

Received: 3 December 2018

Accepted: 2 February 2019

Published online: 26 July 2019

Acta Medica (Hradec Králové) 2019; 62(2): 69-71

https://doi.org/10.14712/18059694.2019.105

(C) 2019 The Authors. This is an open-access article distributed under the terms of the Creative Commons Attribution License (http://creativecommons.org/licenses/by/4.0), which permits unrestricted use, distribution, and reproduction in any medium, provided the original author and source are credited. 


\section{INTRODUCTION}

The recurrent laryngeal nerve (RLN) innervates the intrinsic muscles of the larynx except the cricothyroid. The typical course of RLN is to ascend on either side of the trachea and enter the larynx just lateral to the ligament of Berry. On the right side, the recurrent laryngeal nerve separates from the vagus as it crosses the subclavian artery, passing posteriorly and ascending in a lateral position to the trachea along the tracheoesophageal groove. While on the left side, the recurrent laryngeal nerve separates from the vagus as it traverses over the arch of the aorta.

The nonrecurrent laryngeal nerve (nRLN) is a rare anatomical variation, of the recurrent laryngeal nerve (RLN) that takes an aberrant course and not descending into the thorax (1). The incidence of nRLN in thyroid surgery has been reported from 0.3 to $1 \%$ on the right side, while it is extremely rare on the left side (0.004\%) (2).

The nRLN is consistently related to the absence of brachiocephalic trunk and the presence of the so-called arteria lusoria resulting in an aberrant subclavian artery that runs behind the esophagus on the right side. It only appears on the left side if associated with situs inversus (3). The existence of a nRLN without the associated vascular anomalies has no clear embryological explanation (1).

\section{CASE REPORT}

A 47-year old woman with no known medical history or chronic illnesses presented to our Department with a right-sided thyroid nodule for one year. She had no complaints of hoarseness or compressive symptoms. On examination, the right lobe of the thyroid was found to be enlarged. An indirect laryngoscopic examination showed normal vocal cord function. Ultrasound revealed a solitary, solid $0.82 \times 0.71 \mathrm{~cm}$ nodule in the right lobe. There were no cervical nodes seen on ultrasound. However Calcitonin levels were remarkably high $(22.7 \mathrm{pg} / \mathrm{ml})$. She was confirmed to be euthyroid (TSH $3.2 \mathrm{mU} / \mathrm{l}$ ) and (FT4 $12.0 \mathrm{pg} / \mathrm{ml}$ ). The patient underwent fine needle aspiration and opted for a total thyroidectomy and lymph node dissection ipsilateral and contralateral in central compartment VI. The nerve on the left side was found in the left tracheoesophageal groove in a recurrent fashion, where as in the right side the thyroid lobe was rotated medially and the right laryngeal nerve was not recurrent as it was not found as expected in or near the tracheoesophageal groove. However, careful exploration revealed a transverse nerve running medially to the cricothyroid joint parallel and superficial to the trunk of the inferior thyroid artery (Fig. 1, 2) The nerve was preserved without damage. Histopathological evaluation revealed medullary carcinoma. The postoperative period was uneventful and the patient had no change in her voice. Histopathology revealed two foci of papillary carcinoma $0.3 \mathrm{~cm}$ and $0.1 \mathrm{~cm}$ in the lower pole of the right lobe. Hashimoto thyroiditis was also remarkable. Neoplasia of the parafollicular cells $\mathrm{C}$, with positive Calcitonin antibodies was also revealed. Lymph nodes were infiltrated from papillary carcinoma with immunohistochemistry positive for TTF-1. No infiltration in twelve lymph nodes of the right central compartment and no infiltration in eight yielded lymph nodes of the left central compartment, was found. Follow-up with CT of the chest, MRI of the liver, bone scintigraphy and Calcitonin, CEA levels were within normal range.

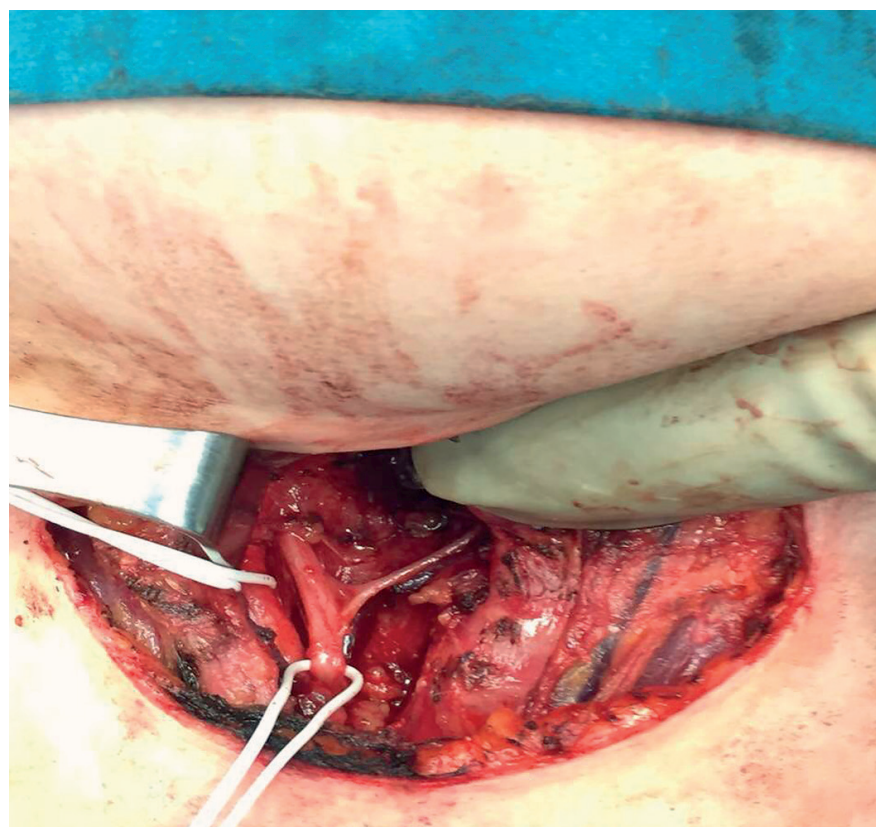

Fig. 1 Right side view of a patient with nRLN during total thyroidectomy. The vagus trunk is medially and superficially located to the common carotid artery.

(CCA: Common Carotid Artery, VN: Vagus Nerve, nRLN: nonRecurrent Laryngeal Nerve, ESO: Esophagus, TR: Trachea)

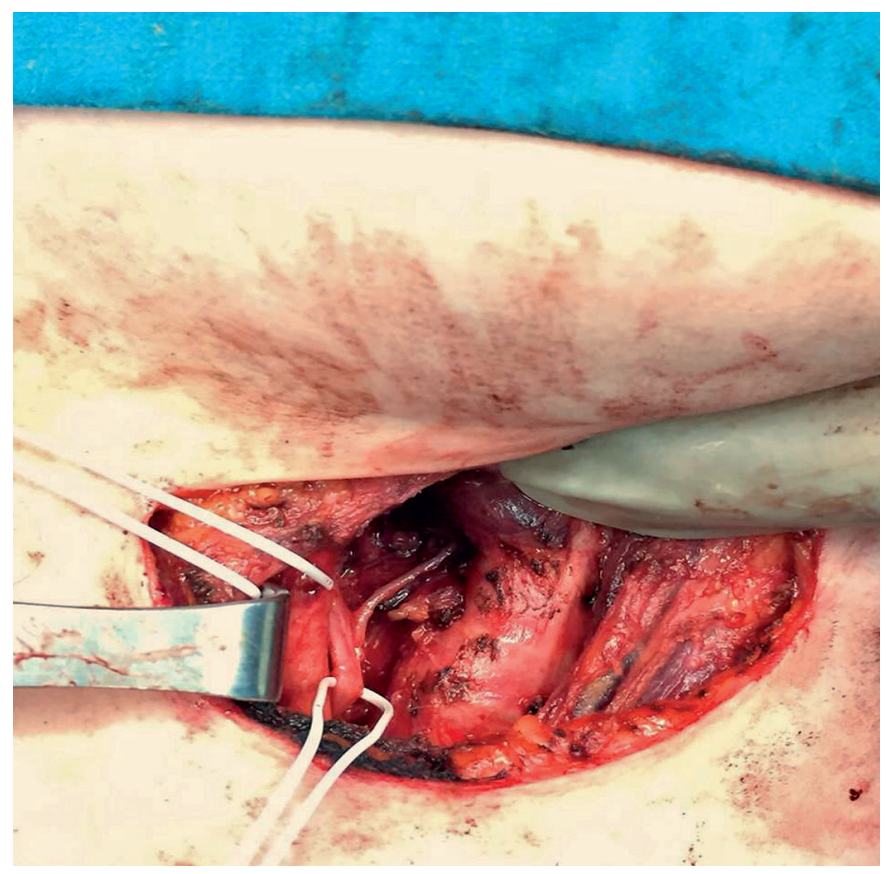

Fig. 2 Right lateral view of a patient undergoing total thyroidectomy. The right non-recurrent laryngeal nerve arises directly from cervical vagus trunk and enters the larynx just parallel to the inferior thyroid artery (Type 2A). The inferior thyroid artery is ligated.

(CCA: Common Carotid Artery, VN: Vagus Nerve, nRLN: nonRecurrent Laryngeal Nerve, CM: Cricopharyngeal Muscle, TR: Trachea) 


\section{DISCUSSION}

The nRLN anatomic variation was first reported by Steadman in 1823, in a cadaver, along with an anomaly of the origin and course of the right subclavian artery (4).

The origin of the nRLN is cervical and there are three types reported (4).

Type 1 occurs when the nRLN arises directly from the vagus and travels with the superior thyroid pedicle vessels. Type 2 is further divided into subtypes A and B.

In Type 2A, the nRLN travels transversely, parallel and superficial to the trunk of the inferior thyroid artery and Type 2B occurs when the nerve travels in a transverse path parallel, but deep to or between the branches of the inferior thyroid artery. Our case can be described as a Type 2A nRLN.

A nRLN can be injured intraoperatively and this may lead to long-term postoperative complications such as vocal cord paralysis. Adequate identification dissection and isolation is very important for preventing damage (1).

In a systematic review by Henry B et al, an overall pooled prevalence of right nRLN of $0.7 \%$ was reported in the general population.

The embryological pathogenesis of an nRLN with an aberrant subclavian artery is clear and understood, but the presence of the variant nerve without the accompanying vascular anomaly, like in our case, remains to be elucidated.

In the literature, Li X et al have dissected 821 recurrent laryngeal nerves, during 2496 thyroid operations, from which 5 were confirmed to be nRLN (0.61\%) (5).

Henry JF et al reported 33 cases of nRLN in 6307 cervicotomies for thyroid and parathyroid operations (0.52\%). The anomaly was observed in 31 cases from 4921 dissections on the right side (0.63\%) and in two cases from 4673 dissections on the left side (0.04\%) (6).

The nRLN was encountered in a study only after performing 585 thyroid operations in three years with an overall incidence of $0.2 \%$ (7). Others have reported a small recurrent laryngeal nerve with a major non-recurrent trunk $(7,8)$.

\section{CONCLUSIONS}

Non-recurrent laryngeal nerve incidence is very rare. When encountered there is a high risk of iatrogenic injury during thyroidectomy or head and neck operations in general. Preoperative diagnosis can only be suspected when there is situs inversus or arteria lusoria. The present case, reports a patient without a vessel anomaly and nRLN was an incidental find during total thyroidectomy for medullary carcinoma.

We decided to present this case because it highlights this anatomical variant. Awareness of its existence and correct surgical technique can minimize the morbidity of iatrogenic damage of the inferior (recurrent or non-recurrent) laryngeal nerve.

\section{CONSENT}

Written informed consent was obtained from the patient for publication of this case report and any accompanying images.

\section{CONFLICT OF INTEREST}

The authors declare no conflict of interest.

\section{REFERENCES}

1. Henry BM, Sanna S, Graves MJ, et al. The Non-Recurrent Laryngeal Nerve: a meta-analysis and clinical considerations. PeerJ 2017; 5: e3012.

2. Kamath S, Rathnakar P, Shetty K. Non-recurrent Laryngeal Nerve: A Rare entity. NUJHS 2012; 2(1).

3. Guerreiro S, Lamas M, Candeias H, Eusébio R, Rocha V. The non-recurrent laryngeal nerve: An anatomical "trap" Rev Port Endocrinol Diabetes Metab 2014; 9: 84-7.

4. Forde R, Williams EW. The Non-recurrent Laryngeal Nerve: A Rare Phenomenon Which Requires Vigilance. West Indian Med J 2015; 64: 303-4.

5. Li X, Wang Z, Lu X, Li J, Huang Y, Huang J, Long X. Non-recurrent laryngeal nerve related to thyroid surgery: a report of 5 cases and literature review. Med Sci Monit 2010; 16(6): CS71-5.

6. Henry JF, Audiffret J, Denizot A, et al. The nonrecurrent inferior laryngeal nerve: review of 33 cases, including two on the left side. Surgery 1988; 104(6): 977-84.

7. Sanders G, Uyeda R, Karlan M. Nonrecurrent inferior lalyngeal nerves and their association with a recurrent branch. The American J Surg 1983; 146: 501-3.

8. Proye CA, Carnaille EM, Goropoulos A. Non-recurrent and recurrent inferior laryngeal nerve. A surgical pitfall in cervical exploration. The Am Surg 1991; 162: 495-6. 